\title{
DISCUSSÃO DOS RETORNOS À ESCALA NOS CONTEXTOS DAS FUNÇÕES DE PRODUÇÃO E DE CUSTO ${ }^{1}$
}

Eliseu Alves ${ }^{2}$

Resumo - O objetivo deste artigo foi expor a teoria de custo de produção de forma rigorosa, mas num nível de matemática correspondente ao uso de derivadas, evitando-se a demonstração de teoremas que tomam muito espaço. O artigo justifica a existência da função custo, apresenta suas propriedades e mostra como ela se relaciona com a função de produção, no sentido de que uma é a dual da outra. Elasticidades parciais e de escala são discutidas nos contextos da função de produção e da função custo. No equilíbrio, demonstra-se que uma é a recíproca da outra. Indica-se que a curva do custo médio não tem forma definida, no sentido de que a forma possa ser deduzida dos axiomas que embasam a teoria da produção, mas salienta-se a plausibilidade da forma em um U aberto. Aduz-se que demanda condicionada a um nível especificado de produção somente existe se a solução do problema de minimização for única. Quando isso ocorre, indicase como obter a função demanda da função custo. Demonstra-se que retorno constante à escala é incompatível com mercados competitivos e que, se prevalecerem, a renda líquida é nula, depois de remunerados todos os fatores de produção. Estende-se a definição da função custo para vários produtos. Enfatiza-se que as planilhas de custo buscam estimar o custo médio mínimo, mas que somente por pura sorte isso ocorre. $\mathrm{O}$ artigo introduz o leitor em textos mais avançados, alguns deles citados no correr da exposição, e é útil para trabalhos econométricos com a função custo.

Palavras-chave: propriedades da função custo, tipos de retorno à escala, função de produção, renda líquida, custo médio.

1 O autor agradece as sugestões de Aloísio Teixeira Gomes, que melhoraram a apresentação do texto. Recebido em: 17/04/2007 Aceito em: 27/06/2007

2 Pesquisador da Embrapa. E-mail: eliseu.alves@embrapa.br 


\section{Introdução}

Fixe-se o nível de produção e imagine-se um experimento em que seja possível ter cem valores diferentes para o preço de cada insumo. Em seguida, calcule-se o custo por unidade de produto e geram-se cem valores diferentes de dispêndios, informalmente de custos de produção. Como o número de informações é finito, igual a cem, sempre existirá o dispêndio mínimo, e ele aproxima o custo de produção para o valor de produção fixado. Entretanto, se for repetido o experimento, muito provavelmente encontrar-se-á outro dispêndio mínimo, e assim sucessivamente, e, desse modo, é possível verificar para qual valor a seqüência converge. Este valor representa o custo mínimo. Na prática, esse procedimento é muito dispendioso, pois assim o é a cada coleta de dados junto aos produtores. O exemplo presta-se, contudo, para ressaltar que o custo de produção é o menor dispêndio, o qual só por pura sorte pode ser obtido numa única tomada de dados, por intermédio de uma planilha.

Então, como obter o custo de produção? Uma das saídas é tentar estimar uma função que se ajuste aos dados coletados de agricultores ou dados de série temporal. Assim, dispõe-se de um conjunto de observações sobre y e w, sendo w o vetor preço dos insumos - como, por exemplo, fertilizante, mão-de-obra, aluguel de terra e de máquinas e equipamentos - e y a produção. Desse modo, cada variação dos preços dos insumos e de y corresponde a um dispêndio. Em geral, pressupõe-se uma forma amena às técnicas econométricas, a qual liga o dispêndio a (w,y). No entanto, sem conhecer as propriedades da função, não há como especificar os sinais dos coeficientes e criticar a forma postulada. É pertinente até duvidar que a função custo exista. Portanto, é importante desenvolver a teoria da função custo para, corretamente, saber ligar dispêndio a (w, y). Outra saída para se determinar o custo é a planilha de custo; mesmo assim, a teoria da função custo ajuda a indicar como deve ser organizada. Entretanto, infelizmente, somente uma informação de dispêndio é obtida para cada observação. 
Embora a teoria da função custo seja complexa, a exposição dela no texto é elementar, e é de interesse do leitor que quer se aprofundar na teoria de produção ou estimar a função custo.

\section{Teoria da função custo: custo total}

A construção da teoria da função custo postula o seguinte: (a) o mercado é competitivo e, por isso, os agricultores seguem, não porque gostem, a regra de comportamento que implica minimizar o custo, ou seja, produzir y com o menor gasto; e (b) conhece a função de produção, $\mathrm{y}=\mathrm{f}(\mathrm{x})$, e os preços dos insumos. Não existe, portanto, incerteza.

Com essas hipóteses, é possível solucionar o problema de minimização do produtor? Ainda não. É necessário admitir que os preços dos insumos sejam maiores que zero e que a função $\mathrm{y}=\mathrm{f}(\mathrm{x})$ seja semicontínua superior, que é uma propriedade menos restritiva que a continuidade. Ela sempre se verifica num conjunto finito de dados. A definição é técnica. É, apenas, enunciada. A função $\mathrm{f}(\mathrm{x})$ é semicontínua superior se o conjunto $A(r)=\{x: f(x) \geq r\}$ for fechado para qualquer número real r. $\mathrm{E} \mathrm{f}(\mathrm{x})$ é semicontínua inferior se o conjunto $A(r)=\{x: f(x) \leq r\}$ for fechado para qualquer número real $\mathrm{r}$. Uma função é contínua se for, simultaneamente, semicontínua superior e inferior.

Admite-se, ainda, que $\mathrm{f}(\mathrm{x})$ satisfaça as seguintes propriedades: $\mathrm{f}(0)=0$; se $x \geq y$, então $f(x) \geq f(y)$, ou seja, é função crescente $^{3}$; se uma componente de $\mathrm{x}$ tender para o infinito $\mathrm{f}(\mathrm{x})$, tende para o infinito, portanto, levanta vôo e contínua subindo. Como é possível ter insumo com preço nulo, será necessário estender a função custo desenvolvida, de modo que comporte também preços nulos. Do ponto de vista da matemática, essa é a parte mais delicada.

${ }_{3} \mathrm{E} x \geq y$ significa que cada componente de $\mathrm{x}$ é maior ou igual à componente correspondente de $\mathrm{y}$. 
O problema de minimização pode ser formulado da seguinte forma:

$$
C(w, y)=\min _{x}\left\{\sum_{i=1}^{n} w_{i} * x_{i}\right\}
$$

Sujeito a:

$$
\begin{aligned}
& y=f(x) \\
& w_{i}>0, i=1, \ldots, n \\
& x_{i} \geq 0, i=1, \ldots, n .
\end{aligned}
$$

O simbolismo quer dizer o seguinte: fixados w e y, procura-se o vetor $\mathrm{x}$ que dá o menor dispêndio, ou seja, a menor soma $\sum_{i=1}^{n} w_{i} * x_{i}$. Se as possibilidades de escolha de $x_{i}$ fossem poucas, obter-se-ia o valor da soma para cada uma delas e se escolheria a menor soma. Mas, como formulado, as possibilidades de escolha dos insumos são muito grandes, infinitas. Por isso, há que se recorrer a métodos mais complexos. A escolha dos insumos não é livre. O vetor escolhido tem que produzir y e não contém componente negativa. Designa-se a solução, se ela for única para todo $(\mathrm{w}, \mathrm{y})$, por $\mathrm{x}(\mathrm{w}, \mathrm{y})$. Assim, $y=f(x(w, y))$ e $C(w, y)=w^{*} x(w, y)$. Note-se que $\mathrm{x}(\mathrm{w}, \mathrm{y})$ não precisa ser único4. Sejam $x^{\prime} e x^{\prime \prime}$ duas soluções. Ora, ambos têm que produzir $\mathbf{y}$, portanto, a solução corresponde a dois pontos da mesma isoquanta e sobre a mesma linha de isocusto, ou seja, $w^{*} x^{\prime}(w, y)=w^{*} x^{\prime \prime}(w, y)$. Logo, mesmo no caso de soluções múltiplas, $C(w, y)$ é bem definida, embora $\mathrm{x}(\mathrm{w}, \mathrm{y})$ não o seja. Desta forma, a cada (w,y) corresponde somente um valor de $\mathrm{C}(\mathrm{w}, \mathrm{y})$, e é esta condição que qualifica $\mathrm{C}(\mathrm{w}, \mathrm{y})$ como função. Qual é o significado de

${ }^{4}$ Neste caso, $X(w, y)$ é uma correspondência. A cada (w, y) corresponde um conjunto de pontos. Essa possibilidade ocorre, por exemplo, quando a isoquanta tem dois pontos ligados por um segmento de reta da mesma inclinação da linha de isocusto. 
$x(w, y)$, no caso de solução única? É a demanda de insumos para produzir $\mathrm{y}$, portanto, a demanda condicional, condicionada por y. Conhecida a função custo e sendo a solução única, como se obtém x(w,y)? Se a função custo tem derivadas parciais em relação a y, então, à derivada em relação ao preço do insumo $\mathbf{i}$ corresponde a demanda condicionada do insumo i. Formalmente, $\partial C(w, y) / \partial w_{i}=x_{i}(w, y)$.

O gráfico a seguir ilustra o procedimento. Nele está a isoquanta y, ou seja, todas combinações dos insumos $x_{1}$ e $x_{2}$ que produzem a mesma quantidade y, e a linha de isocusto, isto é, todas as combinações de $x_{1} \mathrm{e}$ $x_{2}$ que custam a mesma coisa, no caso, $\mathbf{k}$. Para dois insumos:

$$
k=w_{1} * x_{1}+w_{2} * x_{\text {, }}
$$

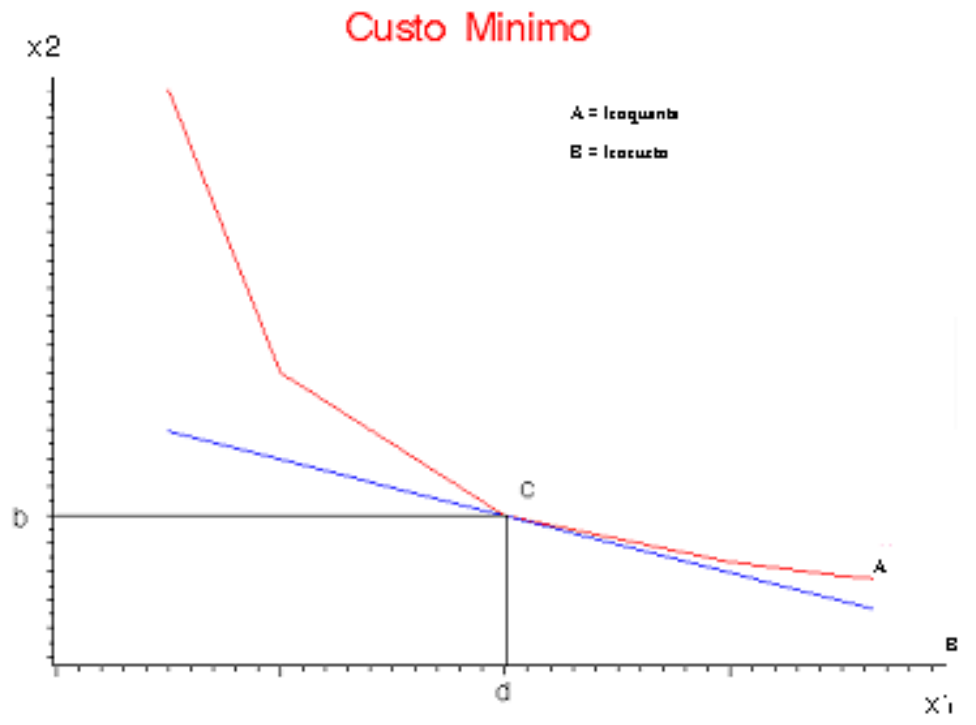


Na solução do problema de minimização, entre as combinações de insumos que produzem y — portanto, se qualificam para a solução uma delas (ou várias delas) tem o menor custo. Quando k aumenta a linha de isocusto, desloca para cima e paralelamente a si mesma. O menor custo é no ponto de tangência da linha de isocusto com a isoquanta. Imagine-se, por um momento, que seja quando a linha de isocusto corta a isoquanta. $\mathrm{O}$ leitor pode verificar que uma linha abaixo da que corta a isoquanta há menor custo, e sempre haverá uma delas, a não ser no caso da tangente. A linha de isocusto abaixo da tangente não tem uma combinação de insumos que produza y. Assim, o mínimo ocorre no ponto de tangência da linha de isocusto com a isoquanta, o ponto $\mathbf{c}$ do gráfico. É possível ter uma isoquanta patológica quando a linha de isocusto corta a isoquanta num ponto e a tangencia noutro. As hipóteses sobre $\mathrm{f}(\mathrm{x})$ visam eliminar as patologias e garantir a existência do mínimo. Todavia, as hipóteses não eliminam a possibilidade de a linha de isocusto tangenciar a isoquanta em mais de um ponto e, mesmo, num número infinito de pontos. Em todos eles, obviamente, o custo é o mesmo, e mínimo. Contudo, a função $\mathrm{x}(\mathrm{w}, \mathrm{y})$, a demanda de insumos, não existirá. Se a função de produção, $y=f(x)$, for estritamente semicôncava, $\mathrm{x}(\mathrm{w}, \mathrm{y})$ será único.

\section{Propriedades da função custo}

Dedica-se mais espaço às propriedades que têm relevância econômica e menos para as de interesse matemático.

1. $C(w, 0)=0$ e $C(w, y)>0$ se $y>0$. Quando nada se produz, nada se gasta; para se produzir alguma coisa, incorre-se em gasto.

2. Para t, número real qualquer, $C(t w, y)=t C(w, y)$, ou seja, se os preços dos insumos dobrarem, o custo dobrará também: $\mathrm{C}(\mathrm{w}, \mathrm{y})$ é linearmente homogênea em w. Seja $C(w, y)=a^{*} w_{1}+b^{*} w_{2}+c^{*} y$. Então, $C(w, y)$ não se qualifica como uma função custo. No caso, se os 
dois preços de insumos, $w_{1} e w_{2}$, forem multiplicados por dois, o custo não dobrará, porque y não fica multiplicado por dois. Assim, uma função custo linear não pode ter o termo y, ou seja, o custo não depende do nível de produção, o que não faz sentido. A função linear não pode ser escolhida para se estimar a função custo. Mas, a função Cobb-Douglas, $C(w, y)=A w_{1}^{a} w_{2}^{b} y$, em que a, b e A são constantes, se qualifica como uma função custo, se $a+b=1$. Caso contrário, não. A função $C(w, y)=A w_{1}^{a} w_{2}^{b} \sqrt{y}$, quando $a+b=1$, é linearmente homogênea. É um exemplo de função custo homotética, que será, posteriormente, discutida.

3. Se os preços de todos ou alguns os insumos aumentarem, e não precisa ser pela mesma quantidade, a função custo não variará ou crescerá. Formalmente, se $w^{1} \geq w^{2}$, então $C\left(w^{1}, y\right) \geq C\left(w^{2}, y\right)$. Ela é crescente em w.

4. Se $y \geq z$, então $C(w, y) \geq C(w, z)$. Para se produzir mais, não se pode gastar menos. Quando isso ocorrer, é porque não se minimizou custo, ou então houve mudança de tecnologia. A propriedade 4 tem sido usada para definir racionalidade e é básica nos métodos que se propõem a estudar a eficiência do agricultor (Varian, 1985; Färe, 1994). Considerem-se, finalmente, as propriedades de interesse matemático.

5. Se y crescer sem limite, então $C(w, y)$ tenderá para o infinito.

6. $\mathrm{C}(\mathrm{w}, \mathrm{y})$ é função côncava em w. Esta propriedade implica ser a matriz hessiana de $\mathrm{C}(\mathrm{w}, \mathrm{y})$, em relação a $\mathrm{w}$, negativa semidefinida ${ }^{5}$.

7. $\mathrm{C}(\mathrm{w}, \mathrm{y})$ é semicontínua inferior em y.

\footnotetext{
Sejam w e h dois vetores preços e $0 \leq t \leq 1$, então $C\left(t^{*} w+(1-t) h, y\right) \geq t^{*} C(w, y)+(1-t)^{*} C(h, y)$.
} 
As propriedades 1 a 7 podem ser demonstradas sem apelo às derivadas. A unicidade da solução exige restrição adicional sobre $\mathrm{f}(\mathrm{x})$. Uma restrição que funciona é ser $\mathrm{f}(\mathrm{x})$ semicôncava estrita. A função $\mathrm{f}(\mathrm{x})$ é semicôncava se o conjunto $A(r)=\{x: f(x) \geq r\}, \mathrm{r}$ um número real qualquer, for convexo ${ }^{6}$. E é semicôncava estrita se $A(r)=\{x: f(x)>r\}$ for um conjunto convexo. Exemplos: a curva na forma de um sino, a curva normal e a linha reta. Toda função côncava é semicôncava. A recíproca não é verdadeira. Quando a função de produção é semicôncava estrita, as derivadas parciais em relação a $\mathbf{w}$ existem, se $y>0$.

\section{Dualidade}

Qual é o significado da palavra dualidade? Verifica-se que, quando a função de produção é semicontínua, é possível obter a função custo como solução do problema de minimização proposto anteriormente. Obtida a função custo, $C(w, y)$, com as propriedades anteriores, é possível recuperar a função de produção que lhe deu origem?

A resposta é positiva, mas exige bastante trabalho, que foge ao nível do texto. Mas, se o problema de minimização tiver uma única solução $\mathrm{x}(\mathrm{w}$, y) e $C(w, y)$ for derivável em relação às componentes de w, a solução é mais simples. Já é conhecido que, neste caso, obtém-se $\partial C(w, y) / d w_{i}=x_{i}(w, y), i=1,2 \ldots n$.Tem-se, assim, um sistema de $\mathrm{n}$ equações em y, w e x, sendo y fixo ${ }^{7}$. Em seguida, busca-se explicitar y em função de $x$. A função obtida é a função de produção. Seja $C(w, y)=A w_{1}^{a} w_{2}^{b} y$ e $a+b=1$, então:

\footnotetext{
Neste caso, a isoquanta, além de ser convexa, não contém nenhum segmento.

$7 \mathrm{~L}(\mathrm{w}, \mathrm{y})=\mathrm{w}_{1}{ }^{*} \mathrm{x}_{1}+\mathrm{w}_{2}{ }^{*} \mathrm{x}_{2}+\ldots+\mathrm{w}_{\mathrm{n}^{\mathrm{x}}} \mathrm{x}_{\mathrm{n}}+\mathrm{q}(\mathrm{y}-\mathrm{f}(\mathrm{x}))$. Derivando-se a função de Lagrange, ao lado, em relação $\mathrm{w}_{\mathrm{i}}$, obtém-se a derivada de $C(w, y)$ em relação $\mathrm{w}_{\mathrm{i} .}$ A função de Lagrange refere-se ao problema de minimização.
} 


$$
\begin{aligned}
& a A w_{1}^{a_{1}-1} w_{2}^{b} y=x_{1} \\
& b A w_{1}^{a} w_{2}^{b-1} y=x_{2}
\end{aligned}
$$

Eliminando $w_{1} e w_{2}$, explicita-se y em função de $x_{1} e x_{2}$ e obtém-se uma função de produção do tipo Cobb-Douglas. Resposta, $y=f(x)=A x_{1}^{a} x_{2}^{b}$ e $A=a^{-a}(1-a)^{a-1}$. Quando a função custo e a função de produção têm a mesma forma, são reciprocamente duais: é o caso da função Cobb-Douglas.

Um exemplo em que dualidade recíproca não ocorre. Para se produzir y são requeridos $n$ insumos e se verifica a seguinte igualdade (função de produção de Leontief):

$x_{i}=a_{i} y i=1,2, \ldots, n$. Então, função custo corresponde a $C(w, y)=\left(\sum_{i=1}^{n} w_{i} * a_{i}\right) * y$. Portanto, a função custo tem forma diferente da função de produção.

\section{Função de produção linear homogênea}

No caso da função de produção Cobb-Douglas, a função custo é o produto de duas funções, uma em $\mathbf{w}$ e a outra em $\mathbf{y}$. É isso verdade para toda função de produção linear homogênea? A resposta é afirmativa, ou seja, sendo a função de produção linear homogênea, então

$C(w, y)=h(w) * y \cdot \mathrm{E} h(w)$ é linear homogênea e côncava em w, como é exigido pelas propriedades 2 , e a recíproca é verdadeira. 
Quando a função de produção é linear homogênea, qual é a inclinação da tangente no ponto em que um raio a partir da origem corta a isoquanta? É a mesma para cada isoquanta. Assim, quando a função é homogênea linear, as isoquantas são paralelas. Seja a função de produção $\mathrm{f}(\mathrm{x})$, em dois insumos (para simplificar) e linear homogênea. Então, $f\left(x_{1}, x_{2}\right)=x_{1} f_{1}\left(x_{1}, x_{2}\right)+x_{2} f_{1}\left(x_{1}, x_{2}\right)$. Ao longo da mesma isoquanta, portanto $f\left(x_{1}, x_{2}\right)$ constante, $f_{1}\left(x_{1}, x_{2}\right) d x_{1}+f_{2}\left(x_{1}, x_{2}\right) d x_{2}=0$. Logo, $\frac{f_{1}\left(x_{1}, x_{2}\right)}{f_{2}\left(x_{1}, x_{2}\right)}=-d\left(x_{2}\right) / d\left(x_{1}\right)$. Ao longo de um raio que passa pela origem e por $\left(x_{1}, x_{2}\right)$, tem-se $x_{1} / x_{2}=K$ e K constante. Derivando $x_{1} / x_{2}=K$, obtém-se $d\left(x_{2}\right) / d\left(x_{1}\right)=\frac{x_{1}}{x_{2}}$. Daí se segue que:

$$
\frac{f_{1}\left(x_{1}, x_{2}\right)}{f_{2}\left(x_{1}, x_{2}\right)}=-d\left(x_{2}\right) / d\left(x_{1}\right)=-\frac{x_{1}}{x_{2}}
$$

Vê-se, desse modo, que, quando se multiplica $x=\left(x_{1}, x_{2}\right)$ por $\mathrm{t}, t \neq 0$, a inclinação da tangente à isoquanta não muda .

\section{Função de produção homotética}

Existe uma função de produção mais geral que a linear homogênea e que tenha esta propriedade? A resposta é afirmativa. Trata-se da função de produção homotética. A função de produção é homotética se for a composição de duas funções. Uma definida nos números reais e monótona crescente estrita e a outra definida no espaço dos insumos e linear homogênea._ $f(x)=r(g(x))$. A função $\mathrm{r}($.) é definida no conjunto dos

\footnotetext{
${ }^{8}$ São $f_{1}(x) e f_{2}(x)$ homogêneas de grau zero. Ao se multiplicar $\mathrm{x}$ por $\mathrm{t}$, as duas derivadas permanecem constantes.
} 
números reais. Quando ela for derivável, exige-se $r^{\prime}(t)>0$. Fazendo $f\left(x_{1}, x_{2}\right)=\mathrm{K}$, Ter-se-á: $r g_{1}\left(x_{1}, x_{2}\right) d x_{1}+r g_{2}\left(x_{1}, x_{2}\right) d x_{2}=0, e d x_{2} / d x_{1}=-g_{1}\left(x_{1}, x_{2}\right) / g_{2}\left(x_{1}, x_{2}\right)$. Ao longo do raio que passa pela origem e por $\left(x_{1}, x_{2}\right),\left(t x_{1}, t x_{2}\right), t>0$, $d x_{2} / d x_{1}=-\frac{x_{2}}{x_{1}}$, como já se viu. Substituindo-se, ocorrerá que:

$\frac{g_{1}(x)}{g_{2}(x)}=-d x_{2} / d x_{1}=-\frac{x_{2}}{x_{1}}$. Ou seja, se $\mathrm{x}$ for multiplicado por $\mathrm{t}, \mathrm{a}$ inclinação da tangente é a mesma. A pergunta relevante é: qual a forma da função custo quando é homotética a função de produção?

$$
C(w, y)=R(y) * h(w) \text { e } R(y)=r^{-1}(y) 9
$$

Novamente, $\mathrm{C}(\mathrm{w}, \mathrm{y})$ desdobra-se em dois fatores: $\mathrm{r}(\mathrm{y})$, monótona crescente estrita em y, e h(w), linear homogênea e côncava em w. Exemplos de função homotética: $C(w, y)=A w_{1}^{a} w_{2}^{b} \sqrt{y}$ e a função translog. Desenvolvê-la é muito trabalhoso (ver Alves, 1996, p. 29-33).

Um ponto importante: se a função de produção é homotética, a função custo se decompõe em dois fatores, como indicado. A recíproca é verdadeira: a decomposição indicada da função custo implica ser homotética a função de produção. E vale o mesmo para a função de produção linear homogênea.

O que ocorre com a demanda condicionada de insumos quando a função de produção é homotética? Decompõe-se no produto de dois fatores: $x_{i}(w, y)=r(y) * \partial h(w) / \partial w_{i}$. Se houver esta decomposição da demanda condicionada de insumo, a função de produção é homotética.

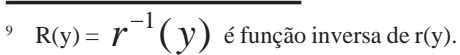


Quando a função de produção é linear homogênea, então $x_{i}(w, y)=y^{*} \partial h(w) / \partial w_{i}$.

Nos logaritmos, $\log \left(x_{i}\right)=\log (y)+\log \left(\partial h(w) / d w_{i}\right)$. Escolhendo-se uma fórmula para $\mathrm{h}(\mathrm{w})$, é possível estimar a equação ao lado. Se ela sobrevier ao teste estatístico, não se rejeitará a hipótese de que os dados são compatíveis com a função de produção linear homogênea.

O grã fico abaixo contém quatro isoquantas e três raios (1,2,3), a partir da origem. Cada raio intercepta as quatro isoquantas em quatro pontos. Seja o raio 1. Quando a função de produção é homotética, as quatro tangentes às isoquantas, nos pontos de intercessão com o raio 1, são retas paralelas. $\mathrm{O}$ mesmo precisa ocorrer com os dois outros raios e qualquer outro raio a partir da origem. Pelo gráfico, visualmente, a função de produção que deu origem a este mesmo é homotética ${ }^{10}$.

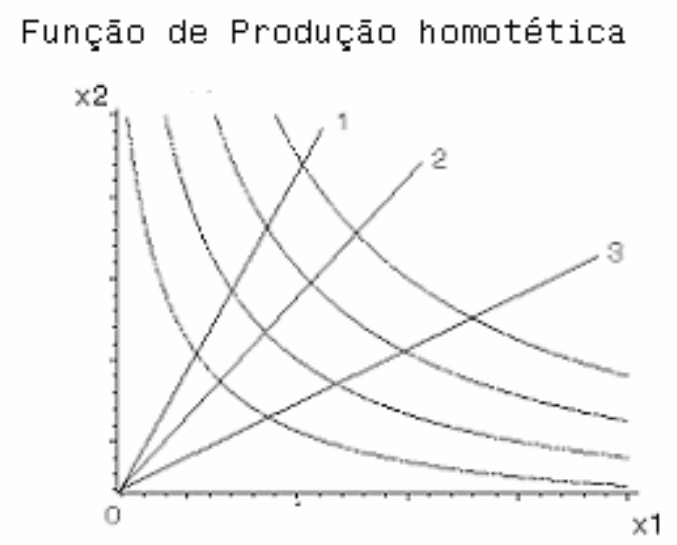

${ }_{10}$ A função de produção linear homogênea é um caso especial de função homotética. Basta, na definição de função homotética, $f(x)=r(g(x))$, tomar $\mathrm{r}()=$.1 . Ou seja, a função $\mathrm{r}$ (.) sempre assume o valor um. 


\section{Produção ótima: curva da oferta}

Em $C(w, y)$, w é mantido constante. Deixa-se variar y para se obter a produção ótima, ou seja, aquela que maximiza a renda líquida.

A renda líquida é dada por $R(w, p, y)=p^{*} y-C(w, y)$, e w e p são constantes, sendo w vetor preço dos insumos e p o preço do produto. Se o máximo de $\mathrm{R}(\mathrm{w}, \mathrm{p}, \mathrm{y})$, em relação a y, existir e o ponto ótimo for tal que $y^{o}>0$, então $p=C^{\prime}(w, y)$. Portanto, o preço do produto é igual ao custo marginal, a derivada do custo $\mathrm{C}(\mathrm{w}, \mathrm{y})$ em relação a $\mathrm{y}$, mantendo-se w fixo. A condição de segunda ordem para um máximo requer que $R^{\prime \prime}\left(y^{o}\right)<0$. Portanto, $\quad C^{\prime \prime}\left(w, y^{o}\right)>0$., ou seja, $\quad C^{\prime \prime}\left(w, y^{o}\right)$ é estritamente convexa em y, no ponto ótimo. O ponto ótimo depende de $\mathrm{w}$ e p. É representado por y(w,p), que é a curva de oferta. Descreve como y varia em relação a p, sendo w fixo. Note-se que é admitido ser única a solução.

Seja $\mathrm{R}(\mathrm{w}, \mathrm{p})$ a renda líquida ótima. Em $y(w, p)$, w contribui para deslocar a curva de oferta para esquerda ou para direita. Que direcionamento há?

O incremento do preço de um insumo tanto pode aumentar, deixar inalterada ou aumentar a produção ótima, isto é, deslocar para a direita, não deslocar ou deslocar para a esquerda a curva da oferta. $\quad \begin{array}{ll}\mathrm{m} \\ \mathrm{m}\end{array}$ fator de produção diz-se normal se $\partial x_{i}(w, y) / \partial y>0$, e diz-se inferior se $\partial x_{i}(w, y) / \partial y<0$. Assim, aumentando-se $\mathrm{y}$, em torno do ótimo, aumenta-se a demanda do fator normal e reduz-se a do inferior. Portanto, a definição está de acordo com a intuição. Se o fator produção for normal, o aumento de seu preço reduz a produção, ou seja, a oferta, e o oposto ocorre com o fator inferior. Novamente, de acordo com a intuição. 
Mantendo w fixo e variando $\mathrm{p}$, produz-se deslocamento ao longo da curva de oferta. Que ocorre com a oferta se p crescer? Sabe-se que, no ponto de equilíbrio, $p=C^{\prime}(w, y(w, p))$. Derivando esta igualdade em relação p, ter-se-á $C "(w, y(w, p) * \partial y(w, p) / \partial p=1$. O primeiro termo do produto à direita é positivo, pela condição de segunda ordem para a maximização da renda líquida. Logo, segue-se que $\partial y(w, p) / \partial p>0$. Assim, o aumento do preço do produto implica o aumento da produção. E a redução do preço do produto implica o inverso.

\section{A curva do custo médio}

A curva do custo médio é dada por $\mathrm{CM}(\mathrm{w}, \mathrm{y})=\mathrm{C}(\mathrm{w}, \mathrm{y}) / \mathrm{y}$, w fixo e $\mathrm{y}>0$ e variável. É usual pressupor-se que tenha a forma de um U e, no caso, a curva do custo marginal a corte no ponto mínimo. As hipóteses feitas não garantem nem a forma em U nem a existência de um único mínimo. A forma de um $\mathbf{U}$ é justificada por fora, portanto, não é deduzida das hipóteses já feitas. Ou seja, supondo-se que, inicialmente, haja recursos ociosos, enquanto estes existirem, o custo médio é decrescente: é o ramo decrescente da curva do custo médio. Exauridos os recursos ociosos, para produzir mais é preciso gastar mais por unidade de produto. Assim, no ponto de exaustão dos recursos ociosos, o mínimo ocorre e a curva do custo médio começa crescer: é o ramo ascendente. Adiciona-se, desse modo, a hipótese dos recursos ociosos e como influenciam a curva de custo médio, mas sem o rigor necessário. Note-se que esta hipótese tem a ver com a função de produção. Ela, por exemplo, rejeita a função de produção tipo Cobb-Douglas, a não ser que esta seja dividida em três ramos: à esquerda do mínimo, com retorno crescente à escala, portanto, maior que um; no mínimo, linear homogênea (retorno à escala igual a um); e à direita, com retorno decrescente, menor que um. Em trabalhos econométricos é estimado um único ramo: o da direita, usualmente. 
No gráfico a seguir estão os três ramos do custo médio: à esquerda do ponto e, o ramo decrescente, que corresponde à elasticidade de escala da função de produção maior que um; o ponto e, o mínimo do custo médio, equivale à elasticidade de escala igual a um; e, finalmente, à direita de e está o ramo crescente, que equivale à elasticidade de escala menor que um, da função de produção. Ainda cabe aduzir que, em y=0, o custo marginal e o custo médio são iguais. Até o ponto e, o custo marginal é menor que o médio; igualam-se novamente em e; e, à direita de e, o custo marginal é maior que o custo médio ${ }^{11}$.

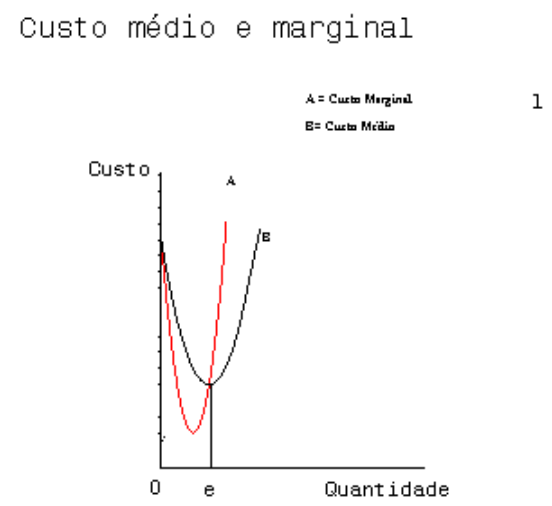

Se a função de produção for linear homogênea, $C(w, y)=y * h(w)$. Logo, o custo médio e o marginal são iguais a $\mathrm{h}(\mathrm{w})$, que é côncava e linear homogênea, em w. Neste caso, a forma em $\mathbf{U}$ do custo médio não se verifica. Ele é uma linha reta paralela ao eixo y, de altura h(w). A linha do custo marginal se justapõe à do custo médio. E a escala de produção não é determinada. Será zero se o preço do produto for menor que $h(w)$ e infinita se maior ou igual. ${ }^{11}$ Pela regra de L'Hospital, $\lim _{x \rightarrow 0}(C(w, y) / y)=C^{\prime}(w, y)$. Por isso, em y $=0$, as duas curvas
coincidem. 


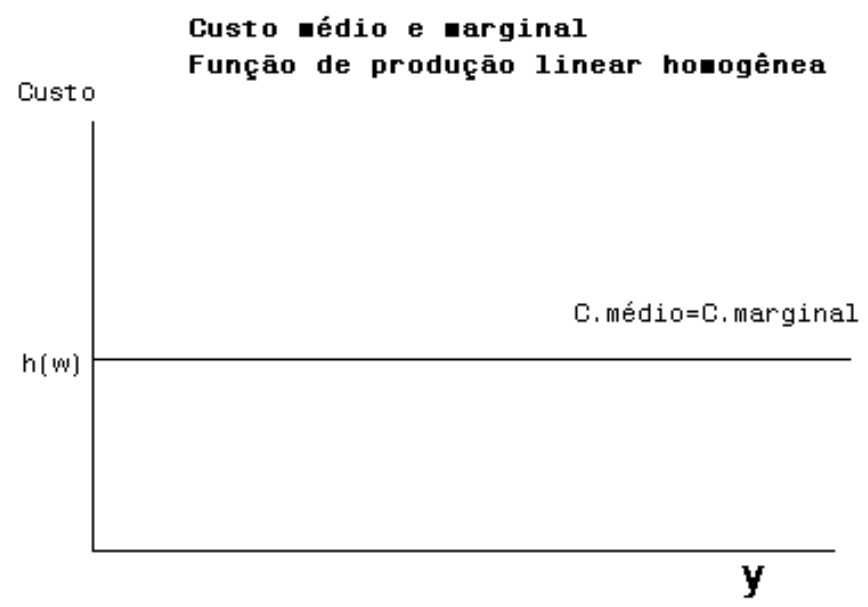

Há duas proposições importantes sobre o impacto do aumento do preço de um insumo na curva de custo médio e na produção: (a) o aumento do preço do insumo $x_{i}$ desloca a curva do custo médio para cima; (b) o incremento do preço do insumo $\mathrm{x}_{\mathrm{i}}$, tudo mais constante, diminui o nível de produção se e somente se $\left(\partial x_{i}(w, y) / \partial y\right) *\left(y / x_{i}\right)>1$. Ou seja, $\partial y(w, y) / \partial w_{i}>0$ se e somente se a desigualdade ao lado se verificar (Alves, 1966, p. 43).

\section{Elasticidades}

Na exposição, restringe-se a dois insumos, porque não há nenhuma dificuldade de se generalizar para n insumos. Há dois tipos de elasticidade de produção: parcial e total.

\section{a. Elasticidade parcial: função de produção}

Seja $y=f\left(x_{1}, x_{2}\right)$. A elasticidade parcial em relação ao insumo $x_{\mathrm{i}}$ indica a porcentagem que a produção crescerá quando se aumenta em $1 \%$ a 
quantidade do insumo i. Formalmente, $e_{i}=\frac{d y / y}{d x_{i} / x_{i}}=f_{i}\left(x_{1}, x_{2}\right) * x_{i} / y, i=1,2$.

Note-se que se fixou $\left(x_{1}, x_{2}\right)$ e, portanto, a elasticidade depende dos níveis de $\left(x_{1}, x_{2}\right)$. No equilíbrio, em que $\left(x_{1}, x_{2}\right)$ correspondem aos valores obtidos pela maximização da renda líquida, tem-se que $p^{*} f_{i}\left(x_{1}, x_{2}\right)=w_{i} \quad i=1,2$. Substituindo, obtém-se $e_{i}=w_{i} x_{i} / p^{*} y=s_{i} \quad i=1,2$.

Ou, generalizando, $e_{i}=w_{i} x_{i} / p^{*} y=s_{i} i=1,2, \ldots, n$. Note-se que $s_{i}$ é a participação do gasto com o insumo i no valor da produção. Reafirmase que essa relação somente é válida no ponto de equilíbrio, que corresponde a p e w.

\section{b. Elasticidade de escala: função de produção}

A elasticidade de escala responde a questão de quanto varia a produção, quando se expandem os insumos, ao longo de um raio que passa pela origem. Ou seja, para $t \geq 0,\left(t x_{1}, t x_{2}\right)$ é o raio a partir da origem e passando por $\left(x_{1}, x_{2}\right)$, quando $t=1$. Por definição, a elasticidade de escala é dada por: $e=\frac{\Delta y / y}{\Delta t / t}$, deixando-se t tender para zero e fazendose $t=1$, ter-se-á $e=\frac{d y}{d t} *(1 / y(1))^{12}$. Derivando $f\left(t x_{1}, t x_{2}\right)$ em relação e para $t=1$, obtém-se $\frac{d y}{d t}=f_{1}\left(x_{1}, x_{2}\right) x_{1}+f_{2}\left(x_{1}, x_{2}\right) x_{2}$. Dividindo ambos os membros da equação por $y>0$ e considerando a definição de elasticidade parcial, tem-se: $e=e_{1}+e_{2}$. Note-se que, para

${ }_{12} y(1)=f\left(x_{1}, x_{2}\right)$. 
cada nível de insumos, $\left(x_{1}, x_{2}\right)$, ao longo do raio que passa pela origem e por $\left(x_{1}, x_{2}\right)$, tem-se um valor de e. Generalizando-se para $\mathrm{n}$ insumos, $e=\sum_{i=1}^{n} e_{i} \cdot$ Na função Cobb-Douglas, os expoentes são as elasticidades parciais, e a elasticidade de escala, pela fórmula acima, é a soma deles. Note-se que, neste caso, as elasticidades parciais e de escala independem de x. Por isso, a função Cobb-Douglas tem elasticidade constante, ou seja, as elasticidades parciais e de escala não dependem de x. Como $e=\sum_{i=1}^{n} e_{i}$, e $e_{i}=s_{i}$, no ponto de equilíbrio, logo, $e=\sum_{i=1}^{n} s_{i}$, no nível ótimo de renda.

Seja a função de produção linear nos dois insumos $x_{1}$ e $x_{2}: y=a+b^{*} x_{1}+c * x_{2}$. Então, $e_{1}=b * x_{1} / y ; e_{2}=c * x_{2} / y$. Portanto, a elasticidade de escala, e, em $\mathrm{x}=\left(x_{1}, x_{2}\right)$, é dada por $e=e_{1}+e_{2}$

Completando a definição de retorno à escala, se, em $\mathrm{x}, e>1$, há retorno crescente à escala; se $e<1$, o retorno à escala é decrescente; e, finalmente, se $e=1$, o retorno à escala é constante.

\section{Retorno à escala: função custo}

A elasticidade de escala é definida da seguinte forma:

$$
E=\frac{\frac{\Delta C(w, y)}{C(w, y)}}{\frac{\Delta y}{y}}=\frac{\Delta C(w, y)}{\Delta y} * \frac{y}{C(w, y)}
$$


É usual deixar $\Delta y$ tender para zero; e levando-se em conta que $d C(w, y) / d y=C^{\prime}(w, y)$, tem-se que $E=C^{\prime}(w, y) *(y / C(w, y))$. Para entender o significado de retorno à escala, no contexto da função custo, convém estudar, rapidamente, a função custo médio, $C M(w, y)=C(w, y) / y$. Derivando CM em relação a y e considerando a definição de $\mathbf{E}$, obtém-se:

$$
C M^{\prime}(w, y)=\frac{\left(y C^{\prime}(w, y)-C(w, y)\right.}{y^{2}}=\left(\frac{C(w, y)}{y^{2}}\right) *\left[C^{\prime}(w, y) * \frac{y}{C(, w y)}-1\right]=\left(\frac{C(w, y)}{y^{2}}\right)(E-1)
$$

É usual admitir ter a curva do custo médio a forma de um $\mathbf{U}$ aberto, com um ponto mínimo. À esquerda do ponto mínimo a curva do custo médio é decrescente; logo, $C M$ ' $(w, y)<0$, o que implica, pelo último membro à direita de $(*)$, que $E<1$. Neste ramo da curva do custo médio predomina o retorno crescente à escala, porque se produz mais com custo decrescente. Quando $E=1$, a curva do custo médio passa por um mínimo (verifique a condição de segunda ordem), tem-se retorno constante à escala. À direita do mínimo, a curva do custo médio é crescente; logo, $C M^{\prime}(w, y)>0$, o que implica ter-se $E>1$. Neste ramo, produz-se mais com custo crescente.

É pertinente reescrever o segundo membro de $(*)$ da seguinte forma:

$$
C M^{\prime}(w, y)=\frac{\left.C^{\prime}(w, y)-C(w, y) / y\right)}{y} \quad(* *)
$$

À direita do ponto mínimo o custo médio é decrescente, portanto, $C M^{\prime}(w, y)<0$, assim, $C^{\prime}(w, y)<C(w, y) / y$. Desse modo, a curva do custo marginal está abaixo da do custo médio. No ponto de custo médio mínimo elas se cruzam. À direita deste ponto, a curva do custo marginal fica acima da curva do custo médio. 


\section{Elasticidades parciais pela função custo}

Pode-se valer de $\mathrm{x}(\mathrm{w}, \mathrm{y})$, a demanda condicionada, para definir as elasticidades parciais:

$$
E_{i}=\frac{d x_{i}(w, y) / x_{i}(w, y)}{d y / y}=\left(\partial x_{i}(w, y) / \partial y\right) *\left(y / x_{i}(w, y)\right)
$$

Note-se que as elasticidades parciais dependem do nível em que y é fixado. Ora, $C(w, y)=\sum_{i=1}^{n} w_{i} * x_{i}(w, y)$. Tendo-se em conta esta equação e, por definição, a elasticidade de escala em y, é igual a $E=\frac{d C(w, y) / C(w, y)}{d y / y}$, vem, depois de manipulações simples, $E=\sum_{i=1}^{n} S_{i} * E_{i}$. Ora, $S_{i}=w_{i} * x_{i}(w, y) / C(w, y)$. Ou seja, $\mathrm{S}_{\mathrm{i}}$ mede a participação do dispêndio no insumo $\mathrm{x}_{\mathrm{i}}$ no dispêndio total, $\mathrm{C}(\mathrm{w}, \mathrm{y})$.

12. Relação das elasticidades nas óticas das funções de produção e de custo

No ponto ótimo, $y_{o}, e=\left(w_{1} x_{1}+w_{2} x_{2}\right) / p y_{0}=C\left(w, y_{o}\right) / p y_{o}$. No ponto ótimo, tem-se $\quad C^{\prime}\left(w, y_{0}\right)=p$. Como $E=C^{\prime}\left(w, y_{0}\right) *\left(y / C\left(w, y_{0}\right)\right), \quad$ no ponto ótimo, $y_{o}, E=p y_{0} / C(w, y)$, comparando $\mathbf{E}$ e e conclui-se que no ponto de renda líquida máxima, $y_{0}$, tem-se $E=1 / e$. No caso da função CobbDouglas, ambas as elasticidades independem do ponto ótimo, verifique se esta relação ocorre. Óbvio, não? 


\section{Renda líquida e retorno constante}

$\mathrm{Na}$ condição de retorno constante, a renda líquida é nula. Lembre-se que, neste caso, as curvas de custo médio e custo marginal coincidem, numa linha paralela ao eixo das quantidades. Se o preço estiver abaixo desta linha, não haverá produção. Se estiver acima, a renda líquida é positiva, mas o crescimento da produção, que virá em conseqüência, fará, com o passar do tempo, que o preço do produto volte para o nível em que a linha referida corta a ordenada. $\operatorname{Logo}, p=C(w, y) / y, y>0$, ou então, $p y=C(w, y)$, e a renda líquida é nula ${ }^{13}$. Como $t R L=t\left(p f(x)-w^{*} x\right) t>0$, no caso de retorno constante, se $R L>0$ para algum $\mathrm{x}, \mathrm{RL}$, renda líquida, crescerá para o infinito, deixando t crescer indefinidamente, o que não faz sentido. Assim, na condição de retorno constante, qualquer nível de produção produz a mesma renda líquida, a qual é zero. O equilíbrio é, portanto, indefinido. Assim, a firma poderia expandir a produção sem limites, tornando-se a única firma produtora, com o domínio total do mercado. Neste caso, a hipótese que fundamentou o exercício - de que o mercado é competitivo - deixaria de ser verdadeira. Ainda, no mundo da agricultura isso não existe. Logo, a hipótese de retorno constante não pode prevalecer no longo prazo.

\section{Produção múltipla}

O sistema de produção pode dar origem a dois ou mais produtos, sem que exista uma função de produção separada para cada produto. No caso de existir uma função de produção para cada produto, volta-se ao caso anterior, e há uma função custo para cada produto. A produção de leite, por exemplo, produz três produtos: leite, vacas que serão descartadas e bezerros; assim, a produção não se separa em três funções de produção. Por isso, é necessário substituir a função de produção pelo conjunto de

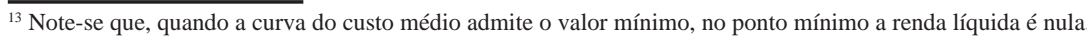


produção $\mathrm{V}(\mathrm{y})$ e $V(y)=\{x: x$ produz $y\}$, e $\mathrm{V}(\mathrm{y})$ é um conjunto fechado. O problema de minimização é formulado da seguinte maneira:

$$
C(w, y)=\min _{x \varepsilon V(y)}\left\{\sum_{i=1}^{n} w_{i}^{*} x_{i}\right\}
$$

Sujeito a:

$w_{i}>0, i=1, \ldots, n$

$x_{i} \geq 0, i=1, \ldots, n$.

As propriedades de $C(w, y)$ são basicamente as mesmas; as derivadas, quando existirem, dizem respeito às componentes dos vetores $\mathrm{w} e \mathrm{y}$. A discussão sobre dualidade é bem mais complicada. A função custo médio existe tão-somente para cada componente do vetor y. É possível definir elasticidade de escala, mas ela perde a interpretação dada, e $C(w, y)$ pode ser usada em trabalhos econométricos, considerando-se as componentes de w e y. No equilíbrio, o custo marginal em relação a cada componente do vetor y se iguala ao respectivo preço. Derivando $R L(y)=p^{*} y-C(w, y)$ em relação a cada componente de y e igualando-se a zero, depois de verificar-se a condição de segunda ordem, virá: $p_{i}=C_{i}^{y}\left(w, y_{0}\right), i=1,2, \ldots, n$, em que $y_{0}$ é o valor de y correspondente ao ótimo e tem $\mathbf{n}$ componentes.

\section{Conclusões}

Procurou-se mostrar as propriedades da função custo e como ela se relaciona com a função de produção que lhe deu origem. O texto é uma introdução ao capítulo 1 da monografia intitulada Função Custo (Alves, 1966). A apresentação é rigorosa, embora o nível de matemática seja elementar. $\mathrm{O}$ texto ajuda o leitor a entender melhor o significado das 
planilhas de custo e mostra a implausibilidade da existência de retorno à escala em mercados competitivos. Se estes existissem, o tamanho do negócio ficaria indefinido: qualquer tamanho redundaria na mesma renda líquida, sempre nula. Muitos justificam a reforma agrária, fundamentados na existência de retorno constante à escala. Contudo, o texto mostrou que a proposição não subsiste quando o mercado é competitivo. A função custo expressa a decisão de minimizar os dispêndios para cada nível de produção. Assim, espera-se que os dados reflitam esta decisão nos modelos econométricos. Outra linha de ataque, que não foi estudada no texto, é verificar se os dados refletem a decisão de minimizar dispêndios (Alves, 2004).

\section{Referências}

ALVES, Eliseu. A função custo. Brasília DF: Embrapa, 1966.

Racionalidade dos agricultores: que dizem os dados? Revista de Economia e Agronegócio, v. 1, n. 4, p. 515-529, out./dez 2003, 2004.

CHAMBERS, Robert G. Applied production analysis: a dual approach. New York: Cambridge University Press, 1994.

FÄRE, Rolf; GROSSKOPF, Shawna; LOVEL, C. A. Production frontiers. New York: Cambridge University Press, 1994.

VARIAN, Hall R. Non-parametric analysis of optimizing behavior with measurement error. Journal of Econometrics, v. 30, p. 445-458, 1985. 


\begin{abstract}
The article deals with the cost function at a mathematical level that only requires knowledge of differentials, but except for that, it keeps rigor at a high level. It only states theorems that require long proofs. The article justifies the existence of the cost function, points out its properties, and shows how it relates with the production function in the sense that one is the dual to the other. The article discusses partial and scale elasticities, both in the context of production and cost functions. Whenever profit is maximized, one is the reciprocal of the other. The cost function has not a defined form in the sense that it can be deduced from the axioms of production theory. But the articles points out the plausibility of the form that resembles an open $\mathbf{U}$. The existence of the demand function for a given level of production requires the unicity of the solution of the minimization problem. Whenever the solution is unique, one indicates how to obtain the demand from the cost function. Constant return to scale is not compatible with a competitive economy, and if it prevails net profit is zero, after all factor of production gets its share. The paper extends the cost function to several products, and emphasizes that only by pure luck the minimum average cost shows up if just one observation is surveyed. The article prepares the reader for a more advanced exposition of the cost function, and the text references some of them, and also is useful for econometric research.
\end{abstract}

Keywords: cost function, production function, return to scale, average cost, profit. 